\title{
Memory Test Performance on Analogous Verbal and Nonverbal Memory Tests in Patients with Frontotemporal Dementia and Alzheimer's Disease
}

\author{
Deanna Baldock Justin B. Miller Gabriel C. Leger Sarah Jane Banks \\ Cleveland Clinic Lou Ruvo Center for Brain Health, Las Vegas, Nev., USA
}

\author{
Key Words \\ Nonverbal memory · Verbal memory · Brief Visuospatial Memory Test · Frontotemporal \\ dementia - Alzheimer's disease
}

\begin{abstract}
Background: Patients with frontotemporal dementia (FTD) typically have initial deficits in language or changes in personality, while the defining characteristic of Alzheimer's disease (AD) is memory impairment. Neuropsychological findings in the two diseases tend to differ, but can be confounded by verbal impairment in FTD impacting performance on memory tests in these patients. Methods: Twenty-seven patients with FTD and 102 patients with AD underwent a neuropsychological assessment before diagnosis. By utilizing analogous versions of a verbal and nonverbal memory test, we demonstrated differences in these two modalities between AD and FTD. Discussion: Better differentiation between AD and FTD is found in a nonverbal memory test, possibly because it eliminates the confounding variable of language deficits found in patients with FTD. These results highlight the importance of nonverbal learning tests with multiple learning trials in diagnostic testing.

\section{Introduction}

Frontotemporal lobar degeneration is quite common and is estimated to be the cause of twenty percent of all dementing disorders [1]. The clinical manifestation of frontotemporal lobar degeneration, frontotemporal dementia (FTD), is characterized by changes in personality, comportment, and language. Most cases of FTD can be divided into one of three categories based on the initial complaint and predominant deficit during the initial phase of the 
Baldock et al.: Memory Test Performance on Analogous Verbal and Nonverbal Memory Tests in Patients with Frontotemporal Dementia and Alzheimer's Disease

disorder: behavioral variant (bvFTD), language variant (lvFTD), or motor syndromes [2, 3]. Informants often report memory impairment or difficulty learning in patients with FTD, but this is most likely due to difficulties with attention [4]. Studies have shown that learning and orientation are unaffected until later in the disease progression [5]. Episodic memory is also spared until later, although mild working memory deficits may be seen earlier [6]. In contrast, the defining characteristic of Alzheimer's disease (AD) is episodic memory impairment [7]. Although other cognitive domains are eventually affected, the earliest declines are seen in learning and retaining new information [8]. Not only do individuals with AD have a poorer rate of learning, acquisition tends to plateau earlier than healthy counterparts [9].

Both FTD and AD patients can have hippocampal atrophy [10] and medial temporal lobe atrophy has been found in patients with AD as well as patients with two types of FTD (semantic dementia and right temporal lobe variant) [7]. However, in both studies, there were significant differences in the cognitive testing between the AD and FTD groups. It has been found that deficits in orientation are more predictive of $\mathrm{AD}$, whereas deficits in problem solving are more strongly associated with FTD [11]. Using the National Alzheimer's Coordinating Center (NACC) database, Leger and Banks [3] showed that clinically diagnosed FTD patients with AD pathology at autopsy were less likely to have personality change, problem solving deficits, or reduced judgment as an initial symptom. Patients clinically diagnosed with bvFTD, but who actually had AD findings at autopsy, were more likely to have early memory deficits than those with FTD pathology at autopsy. However, the memory test reported in the NACC database, as part of the Uniform Data Set, is the first story of the Wechsler Memory Scale Logical Memory subtest, a verbally mediated test. This test may be vulnerable to poor scores in bvFTD due to language problems or other executive deficits and may not be the best test of memory in this population.

Memory testing can be categorized by the modality of the stimuli used: verbal or nonverbal. Nonverbal memory testing is usually visuospatial in nature. Nonverbal memory tests have previously been used in studies with FTD patients. Pleizier et al. [7] found that patients with two types of FTD (semantic dementia and right temporal atrophy) perform significantly better at a memory test than AD patients when using a test that is predominantly visual (the Visual Association Test), but it has a verbal component. Frisoni et al. [10] found that verbal and nonverbal recall is better in FTD patients than AD patients, with nonverbal being a more pronounced difference. This difference was seen even though the study excluded patients with primary progressive aphasia. Thus, nonverbal memory tests may be more accurate measures of memory in FTD. Pennington et al. [12] found that patients with bvFTD did not differ from patients with FTD; however, they used a verbal memory test and a nonverbal test that only had one learning trial.

Assuming that it is best to discriminate cognitively between FTD and AD by memory tests, and language and attention are both frequently impacted in FTD [13], choosing memory tests that avoid excessive demands on language or attention may be important for diagnostic purposes. Additionally, tests with only one learning trial (e.g., Rey-Osterrieth Complex Figure Test, Visual Reproduction) can be greatly impacted by a lapse in attention [14]. Furthermore, multiple learning trials with free recall trials are the most sensitive for identifying the memory deficit in $\mathrm{AD}$ [9]. Therefore, we used an analogous pair of verbal and nonverbal memory tests: the Hopkins Verbal Learning Test-Revised [15] (HVLT-R) and the Brief Visuospatial Memory Test-Revised [14] (BVMT-R). Both have three learning trials, a delayed recall trial, and a recognition trial. We hypothesized that the nonverbal test would be especially helpful in distinguishing between FTD and AD patients in clinic. We further expected that the components of these tests that truly measure episodic learning (i.e., delayed recall trials) would be more helpful in distinguishing FTD and AD because episodic memory is impacted early in the $\mathrm{AD}$ process. 
Baldock et al.: Memory Test Performance on Analogous Verbal and Nonverbal Memory Tests in Patients with Frontotemporal Dementia and Alzheimer's Disease

Table 1. Demographic and disease severity data for the two groups

\begin{tabular}{lcl}
\hline & FTD & AD \\
\hline Age, years* & $68.23(9.1)$ & $72.48(9.4)$ \\
Education, years & $13.95(2.8)$ & $14.59(3.1)$ \\
BNT (max. 60) & $37.6(19.6)$ & $44.99(11.2)$ \\
MoCA (max. 30) & $17.19(5.0)$ & $16.98(4.7)$ \\
ADLQ, \% & $29.4(19.5)$ & $34.7(18.2)$ \\
\hline
\end{tabular}

* Statistically significant difference $(\mathrm{p}=0.05)$.

\section{Methods}

\section{Participants}

All methods were approved by the Cleveland Clinic Institutional Review Board. Patients gave their consent for their clinical data to be used in future research. Patients were consecutive referrals seen in our neuropsychology clinic as part of routine clinical care in a specialized center for neurodegenerative disease. Diagnoses were made by the referring neurologist after the neuropsychological assessment. Diagnoses by neurologists were made using current research diagnostic criteria $[16,17]$ and based on clinical history, neurological examination, and neuropsychological assessment, as well as imaging and available biomarker data (e.g., $\mathrm{CSF}$, genetic testing in rare cases). Out of the neurodegenerative disorders clinic, we included a clinical sample in the current study of all cases that met the inclusion criteria. The inclusion criteria were simply if a patient was diagnosed with AD or FTD that had a neuropsychological workup by our clinic in the past. We included 27 patients with FTD, later further subdivided into the behavioral variant $(n=14)$ or language variant $(n=13)$. Patients with motor variants of FTD were excluded as well as patients considered for possible logopenic dementia. A group of 102 patients with probable AD were used for comparison. Table 1 includes demographic information and supporting cognitive data for the FTD and AD groups.

\section{Measures}

All patients underwent a standard battery of tests given in our clinical neuropsychology service. Testing is administered by a neuropsychology technician under the supervision of a neuropsychologist. This 2-hour battery includes tests of memory, attention, executive functioning, visuospatial functioning, attention/processing speed, and language (including the Boston Naming Test [18], BNT). Caregiver questionnaires are also administered to collect information on neuropsychiatric symptoms and activities of daily living using the Activities of Daily Living Questionnaire [19] (ADLQ). In addition, all patients had previously been given a cognitive screen, the Montreal Cognitive Assessment [20] (MoCA), at their initial appointment with the neurologist. The MoCA is a quick cognitive assessment for initial screening that briefly (usually in less than $10 \mathrm{~min}$ ) tests memory, language, orientation, visuospatial abilities, etc. The MoCA gives the neurologist a snapshot of cognitive functioning.

As part of memory assessment in the standardized clinical neuropsychology battery, patients are administered the HVLT-R and the BVMT-R. Both tests have three learning trials (with an immediate recall after each learning trial), one delayed recall trial after a 20-25 min delay, and one recognition trial immediately following the delayed recall trial. The HVLT-R asks participants to learn and recall twelve words, in any order, whereas the BVMT-R asks participants to learn and recall six nontraditional shapes, with one point awarded for replicating the shape reasonably accurately and one point given for recalling its location on the page. The BVMT-R also has a final trial where the patient is asked to simply copy the figures 
Table 2. Results of repeated measures ANOVA demonstrating results and corresponding effect sizes

\begin{tabular}{|c|c|c|c|c|c|c|}
\hline \multirow[t]{2}{*}{ Comparison } & \multicolumn{2}{|c|}{ Main effect group } & \multicolumn{2}{|c|}{ Main effect test } & \multicolumn{2}{|c|}{ Test-group interaction } \\
\hline & $\mathrm{F}$ & $\mathrm{p}\left(\mathrm{eta}^{2}\right)$ & $\mathrm{F}$ & $\mathrm{p}\left(\mathrm{eta}^{2}\right)$ & $\mathrm{F}$ & $\mathrm{p}\left(\mathrm{eta}^{2}\right)$ \\
\hline Trial 1 & 8.36 & $0.005(0.07)^{*}$ & 9.48 & $0.003(0.07)^{* *}$ & 8.72 & $0.004(0.07)^{* *}$ \\
\hline Trial 3 & 10.16 & $0.002(0.08)^{* *}$ & 15.32 & $<0.0005(0.11)^{* *}$ & 8.12 & $0.005(0.06)^{*}$ \\
\hline Delayed & 26.73 & $<0.0005(0.18)^{* *}$ & 6.14 & $0.015(0.05)^{*}$ & 5.70 & $0.019(0.04)^{*}$ \\
\hline Recognition & 4.79 & $0.03(0.04)^{*}$ & 3.01 & $0.09(0.03)$ & 2.92 & $0.09(0.03)$ \\
\hline
\end{tabular}

* and light gray shading correspond to $\mathrm{p}<0.05$; ** and dark gray shading correspond to $\mathrm{p}<0.005$.

in front of them, with no time restriction, and therefore has no episodic memory component. This allows for detection of any primary visuospatial or visuoconstructional problems that might restrict the patient's ability to perform well on the memory tests. The BNT is a test where the patient is shown a series of pictures and asked to produce the correct name for each one. The ADLQ is a questionnaire completed by a close friend or family member that produces a quantified score for the patient's successful functioning in regards to taking care of themselves. In the ADLQ, each test item has options that describe functioning in various areas of life and the higher scores indicate independence so that the score can be converted into a percentage of independence if you place total score of selected items over total points possible.

\section{Analysis}

Demographic data, as well as scores on the BNT, MoCA, and ADLQ were compared with $\mathrm{T}$ tests. Memory test data were first corrected to $\mathrm{Z}$ scores based on within-group mean and standard deviations to ensure that we were comparing scores from the same test on the same scale. We avoided use of norm-based Z scores due to the age differences in our groups; this would have added a confounder of the AD patients being compared with different normative samples than the FTD patients. Once this step was completed, groups were compared via repeated-measures ANOVA on the following scores from each of the two measures: trial 1, trial 3, delayed recall and recognition discrimination (seen in table 2).

\section{Results}

Patients were of a similar level of impairment (mild/moderate) with regard to both independence in activities of daily living and overall cognition, as assessed by the ADLQ and MoCA. Ability to copy figures also did not differ, as based on comparison of the BVMT-R copy trial. Confrontation naming scores also did not differ significantly, as assessed by the BNT. Although language deficits are a key feature of FTD, language deficits are also common in AD, so it is not too unexpected that we did not find a significant difference in BNT scores between the two groups. One possible explanation is that the impaired BNT scores in FTD patients may be due to a purely linguistic deficit. However, the impaired BNT scores in AD patient could be due to a memory impairment during confrontation naming or a mixture of amnestic deficits in combination with language deficits. The only significant difference was age; however, age is an expected difference between the two disorders. Mean scores on BVMT-R and HVLT-R trials were below normal for most patients. The distribution of scores is shown in figures 1-3. The maximum score on each trial is 12 . 
Baldock et al.: Memory Test Performance on Analogous Verbal and Nonverbal Memory Tests in Patients with Frontotemporal Dementia and Alzheimer's Disease

Fig. 1. Mean scores on learning trial 1 for each task, by diagnostic group. Error bars represent one standard deviation.

Fig. 2. Mean scores on learning trial 3 for each task, by diagnostic group. Error bars represent one standard deviation.

Fig. 3. Mean scores on delayed recall trial for each task, by diagnostic group. Error bars represent one standard deviation.
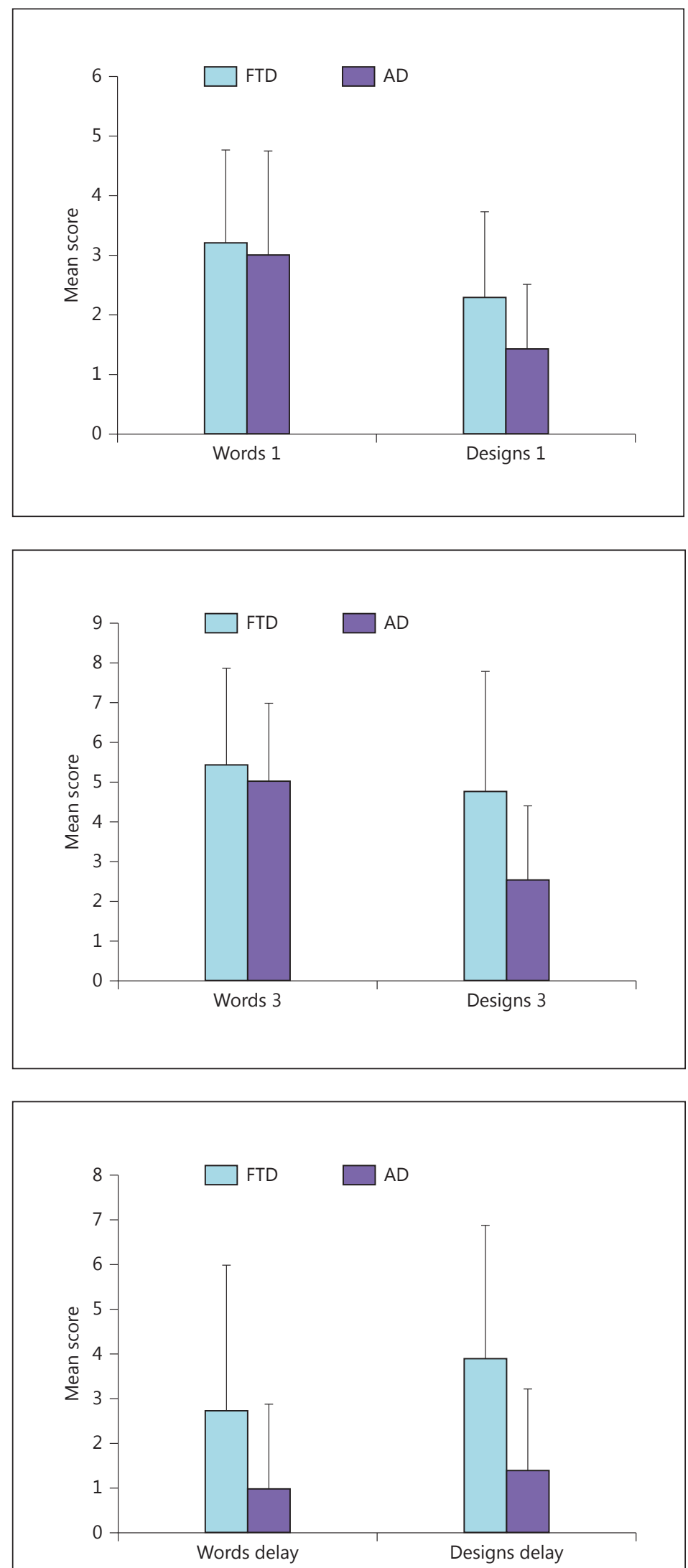
Baldock et al.: Memory Test Performance on Analogous Verbal and Nonverbal Memory Tests in Patients with Frontotemporal Dementia and Alzheimer's Disease

All measures differed between groups, with FTD patients faring better on all aspects of both memory tests. There were main effects of test also, although these are interpreted with caution since they are measured on slightly different scales (i.e., the HVLT-R is number of words learned, while the BVMT-R is a combination of recalling the location and accuracy of reproduction). The overall pattern was that the learning trials were scored more highly on the HVLT-R, and the delayed trial on the BVMT-R across groups. All tests showed a group-bytest interaction with a greater difference in performance for the BVMT-R compared with the HVLT-R in the FTD group specifically. Given the multiple comparisons involved, we are most confident in the differences between groups on trial 3 and the delayed trial where effect sizes were medium to large. We did a similar analysis comparing subgroups of FTD patients (language and behavioral variant); however, this yielded no significant group differences.

\section{Discussion}

This study compared performance on analogous tests of verbal and nonverbal memory between patients with clinically diagnosed FTD and AD. The findings are summarized below.

On the learning trials, both groups scored higher on the verbal than the nonverbal task. We found significant group differences on the third learning trial and the delayed recall task. These differences demonstrated medium to large effect sizes, which is especially important since we calculated multiple comparisons from this data set. The difference between groups on the learning trials and delayed recall is consistent with our prediction, given the known deficit in learning and memory in AD, which is generally not seen in FTD. AD patients showed a more pronounced deficit on learning on the design task compared with the verbal task. FTD patients showed more intact delayed recall of designs (compared with words) in comparison with $\mathrm{AD}$ patients.

These results point to the importance of nonverbal memory testing in distinguishing groups with different forms of neurodegenerative disease, as the nonverbal memory test was the most able to differentiate between FTD and AD. Less discrepancy would be noticed between groups if verbal memory measures were used alone, especially if there was only one learning trial.

Interestingly, there were no significant differences between lvFTD and bvFTD. This could be due to the fact that patients who were too aphasic to complete testing were removed from analysis, or due to insufficient power because of the small sample size, or due to the overlap often seen in bvFTD and lvFTD.

Some previous studies have failed to show significant differences in memory performance between patients with AD and patients with FTD. This could be the consequence of using only a verbal memory task, a task which requires much attention, a task which has a strong executive functioning component, a task which uses only one learning trial, or due to insufficient power. Also, FTDs are somewhat rare and therefore, sample sizes tend to be small, which may result in underpowered analysis in many studies. The study by Frisoni et al. [10] had 44 patients with AD, but only 9 patients with FTD completed the neuropsychological testing. In that study, FTD patients differed significantly from the patients with moderate AD in a task of verbal learning, but did not differ significantly from the patients with mild AD. There was also greater difference between FTD and AD groups in nonverbal delayed recall than verbal delayed recall, but it was less significant than the difference we found. One explanation could be that Frisoni et al. [10] used only one learning trial, whereas the multiple learning trials used in this study reduced the effect of inattention in the patients with FTD and allowed them to encode the information, ensuring better recall scores. As possible evidence of this idea, Thompson et al. [21] found that the disturbance of the frontal lobe (which leads 
to impulsivity, inattention, etc.) impacts test performance in individuals with FTD. They found that individuals with AD had lower test scores than individuals with FTD on memory tests and drawing tests. However, FTD participants also had issues in memory testing, but it seemed related to confabulation and misconstruction. Participants with FTD also had some complications in drawing tests, but it appeared to be due to organizational errors.

Limitations of this study include the potential tautology of using the neuropsychological measures which also contributed to diagnosis as an outcome measure. Future studies may avoid this pitfall by using a priori research methodology where diagnosis is made independent of neuropsychological assessment. Similarly, we categorized our patients clinically; there was no uniform pathological verification of our diagnostic groups. A comprehensive neurological workup was done for each patient, but since there was much variation as to what type of imaging, we did not include imaging results in this study. Large pathologically verified datasets such as the NACC do not, unfortunately, include nonverbal memory measures, and are therefore not suitable for probing this question. Future studies may consider visuospatial dysfunction as a potential restriction in performance on tasks such as the BVMT-R. The availability of amyloid imaging might allow future studies to capitalize on in vivo diagnosis of $A D$ versus FTD with more certainty than clinical judgment. Finally, our participants were fairly well educated, impacting the generalizability of our results.

This study paves the way for numerous other potential investigations. We intend to assess the underlying neuroimaging correlations of our findings: since hippocampal volume correlates with memory performance in AD patients, would temporal or frontal atrophy correspond to poor list learning performance in FTD patients? We would also like to examine differences between patients with FTD, logopenic type AD, and frontal type AD in nonverbal memory tests: are logopenic $\mathrm{AD}$ and frontal $\mathrm{AD}$ more similar to traditional $\mathrm{AD}$ in cognitive testing or does it mirror FTD?

This paper reinforces the need to use multimodal strategies in assessing memory when assessing differential diagnoses. Overall, our findings highlight the potential utility of multiple learning trials of nonverbal information followed by nonverbal memory assessment in diagnostic differentiation between AD and FTD. Given the widespread use of cognitive screening instruments which tend to probe memory using short word lists only, there may be a lack of specificity of many of these measures when trying to differentiate between AD and FTD. Therefore, establishing intact nonverbal memory may, with further research, be an important way to differentiate between these two diseases.

\section{References}

1 Laforce R: Behavioral and language variants of frontotemporal dementia: a review of key symptoms. Clin Neurol Neurosurg 2013;115:2405-2410.

-2 Hodges JR, Davies RR, Xuereb JH, Casey B, Broe M, Bak TH, Kril JJ, Halliday GM: Clinicopathological correlates in frontotemporal dementia. Ann Neurol 2004;56:399-406.

-3 Leger GC, Banks SJ: Neuropsychiatric symptom profile differs based on pathology in patients with clinically diagnosed behavioral variant frontotemporal dementia. Dement Geriatr Cogn Disord 2014;37:104-112.

-4 Barber R, Snowden JS, Craufurd D: Frontotemporal dementia and Alzheimer's disease: retrospective differentiation using information from informants. J Neurol Neurosurg Psychiatry 1995;59:61-70.

5 Frisoni GB, Laakso MP, Beltramello A, Geroldi C, Bianchetti A, Soininen H, Trabucchi M: Hippocampal and entorhinal cortex atrophy in frontotemporal dementia and Alzheimer's disease. Neurology 1999;52:91-100.

6 Rabinovici GD, Miller BL: Frontotemporal lobar degeneration: epidemiology, pathophysiology, diagnosis and management. CNS Drugs 2010;24:375-398.

-7 Pleizier CM, van der Viles AE, Koedam E, Koene T, Barkhof F, van der Flier WM, Scheltens P, Pijnenburg Y: Episodic memory and the medial temporal lobe: not all it seems. Evidence from the temporal variants of frontotemporal dementia. J Neurol Neurosurg Psychiatry 2012;83:1145-1148.

8 Weintraub S, Wicklund AH, Salmon DP: The neuropsychological profile of Alzheimer disease. Cold Spring Harb Perspect Med 2012;2:a006171. 
-9 Petersen R, Smith G, Ivinik R, Kokmen E, Tangalos E: Memory function in very early Alzheimer's disease. Neurology 1994;44:867-872.

10 Frisoni GB, Beltramello A, Geroldi C, Weiss C, Bianchetti A, Trabucchi M: Brain atrophy in frontotemporal dementia. J Neurol Neurosurg Psychiatry 1996;61:157-165.

-11 Varma AR, Snowden JS, Lloyd JJ, Talbot PR, Mann DMA, Neary D: Evaluation of the NINCDS-ADRDA criteria in the differentiation of Alzheimer's disease and frontotemporal dementia. J Neurol Neurosurg Psychiatry 1999; 66:184-188.

12 Pennington C, Hodges JR, Hornberger M: Neural correlates of episodic memory in behavioral variant frontotemporal dementia. J Alzheimers Dis 2011;24:261-268.

13 Neary D, Snowden JS, Gustafson L, Passant U, Stuss D, Black S, Freedman M, Kertesz A, Robert PH, Albert M, Boone K, Miller BL, Cummings J, Benson DF: Frontotemporal lobar degeneration: a consensus on clinical diagnostic criteria. Neurology 1998;51:1546-1554.

14 Benedict RHB: Brief Visuospatial Test - Revised; Professional Manual. Lutz, PAR, 1997.

15 Brant J, Benedict RHB: Hopkins Verbal Learning Test - Revised; Professional Manual. Lutz, PAR, 2001.

16 Dubois B, Gauthier S, Cummings J: The utility of the new research diagnostic criteria for Alzheimer's disease. Int Psychogeriatr 2013;25:175-177.

17 Rascovsky K, Hodges JR, Knopman D, Mendez MF, Kramer JH, Neuhaus J, van Swieten JC, Seelaar H, Dopper EGP, Onyike CU, Hillis AE, Josephs KA, Boeve BF, Kertesz A, Seeley WW, Rankin KP, Johnson JK, Gorno-Tempini ML, Rosen H, Prioleau-Latham CE, Lee A, Kipps CM, Lillo P, Piguet O, Rohrer JD, Rossor MN, Warren JD, Fox NC, Galasko D, Salmon DP, Black SE, Mesulam M, Weintraub S, Dickerson BC, Diehl-Schmid J, Pasquier F, Deramecourt V, Lebert F, Pijnenburg Y, Chow TW, Manes F, Grafman J, Cappa SF, Freedman M, Grossman M, Miller BL: Sensitivity of revised diagnostic criteria for the behavioural variant of frontotemporal dementia. Brain 2011; 134:2456-2477.

18 Kaplan EF, Goodglass H, Weintraub S: Boston Naming Test, ed 2. Philadelphia, Lippincott Williams and Wilkins, 2001.

19 Johnson N, Barion A, Rademaker A, Rehkemper G, Weintraub S: The Activities of Daily Living Questionnaire: a validation study in patients with dementia. Alzheimer Dis Assoc Disord 2004;18:223-230.

20 Nasreddine ZS, Phillips NA, Bedirian V, Charbonneau S, Whitehead V, Collin I, Cummings JL, Chertkow H: The Montreal Cognitive Assessment, MoCA: a brief screening tool for mild cognitive impairment. J Am Geriatr Soc 2005;53:695-699.

21 Thompson JC, Stopford CL, Snowden JS, Neary D: Qualitative neuropsychological performance characteristics in frontotemporal dementia and Alzheimer's disease. J Neurol Neurosurg Psychiatry 2005;76:920-927. 